\title{
The equivariant Conley index and bifurcations of periodic solutions of Hamiltonian systems
}

\author{
ANDREAS FLOER AND EDUARD ZEHNDER \\ Courant Institute of Mathematical Sciences, 251 Mercer Street, New York, \\ NY 10012, USA; Mathematik, ETH-Zentrum, CH-8092 Zürich, Switzerland
}

Abstract. An equivariant version of Conley's homotopy index theory for flows is described and used to find periodic solutions of a Hamiltonian system locally near an equilibrium point which is at resonance.

\section{Introduction}

The aim of this paper is to give an alternative proof of an existence theorem for periodic solutions of Hamiltonian equations, which is due to Fadell and Rabinowitz [1]. It is based on an equivariant version of Conley's homotopy index theory for flows. This theory replaces the mini-max arguments based on a cohomological index theory for Lie group actions used in the original proof.

First, in order to recall the theorem, we consider a Hamiltonian vector field

$$
\dot{x}=J \nabla H(x), \quad x \in \mathbb{R}^{2 n},
$$

where $H \in C^{2}$ is the Hamiltonian function and $J \in \mathscr{L}\left(\mathbb{R}^{2 n}\right)$ is the standard symplectic structure. The aim is to find periodic solutions locally in a neighbourhood of an equilibrium point, which we assume to be the origin $0 \in \mathbb{R}^{2 n}$, such that $\nabla H(0)=0$; we may also assume that $H(0)=0$. We are therefore dealing with a local problem; we search for small periodic solutions in a neighbourhood of the origin assuming only some knowledge of the linearized vector field at the equilibrium point:

$$
\dot{x}=A x, \quad A=J H_{x x}(0) .
$$

Clearly the occurrence of purely imaginary eigenvalues of $A$ is a necessary condition, since otherwise the equilibrium point is hyperbolic and hence excludes small periodic solutions.

If, for example, $\alpha_{1}=i \omega, \alpha_{2}=-i \omega$ for some $\omega \neq 0$ is a pair of imaginary eigenvalues with eigenvectors $A\left(e_{1}+i e_{2}\right)=i \omega\left(e_{1}+i e_{2}\right)$, then (1.2) has the family $x(t):=$ $\operatorname{Re}\left\{c\left(e_{1}+i e_{2}\right) e^{i \omega t}\right\}$ of periodic solutions with period $T=2 \pi / \omega$, which fill out the two-dimensional plane $E=\operatorname{span}\left\{e_{1}, e_{2}\right\}$. A well known result due to Lyapunov [6] guarantees periodic solutions of (1.1) close to these: if the plane $E$ contains all the periodic solutions of (1.2) with period $T$, then for every small $\varepsilon$ the energy surface $H(x)=\varepsilon$ carries a periodic solution whose period is close to $T$. If there are several pairs of imaginary eigenvalues of $A$, one finds several corresponding families of periodic solutions for (1.1) having their periods close to the normal modes, provided 
that the eigenvalues satisfy appropriate non-resonance conditions. If these conditions are not met, the Hamiltonian system may in fact have the equilibrium point as the only periodic solution, as examples show.

It was relatively recently that Weinstein [10] discovered that the non-resonance condition can be replaced by another condition on $A$. To describe it we recall Moser's generalization [7] of his result and assume that

$$
\mathbb{R}^{2 n}=E \oplus F
$$

splits into two invariant subspaces under $A$ such that all solutions of (1.2) in $E$ have period $T>0$ while none of the solutions in $F \backslash\{0\}$ has this period. If now

$$
H_{x x}(0) \mid E>0,
$$

i.e. is definite, then for sufficiently small $\varepsilon>0$ every energy surface $H(x)=\varepsilon$ contains at least $\frac{1}{2} \operatorname{dim} E$ periodic solutions having periods close to $T$.

Observe that no non-resonance conditions are required, but instead $H_{x x}(0)$ is required to be definite on $E$. In the following we are interested in the situation where (1.4) fails, in which case $H_{x x}(0) \mid E$ is not definite. Assuming it is nondegenerate, we abbreviate the signature of the Hessian by

$$
\text { signature } H_{x x}(0) \mid E=2 \sigma \text {; }
$$

it is always an even integer. In this case there are symplectic coordinates in the symplectic subspace $E$ such that the quadratic part $H^{2}$ of the Hamiltonian has the form

$$
H^{2} \mid E=\sum_{j=1}^{n_{+}} \omega_{j} I_{j}-\sum_{j=n_{+}+1}^{n_{+}^{++n}} \omega_{j} I_{j},
$$

with $I_{j}=\frac{1}{2}\left(p_{j}^{2}+q_{j}^{2}\right)$ for $1 \leq j \leq n_{+}+n_{-}$. Moreover, $\operatorname{dim} E=2 n_{+}+2 n_{-},|\sigma|=\left|n_{+}-n_{-}\right|$ and

$$
k_{j} \omega_{j}=2 \pi / T>0 \quad \text { for } 1 \leq j \leq \frac{1}{2} \operatorname{dim} E
$$

with integers $k_{j}>0$. Examples show [8] that the Hamiltonian system (1.1) may have no periodic solutions except the trivial solution in the case $\sigma=0$, i.e. $n_{+}=n_{-}$. In contrast, Fadell and Rabinowitz establish in the case $\sigma \neq 0$ at least $|\sigma|$ periodic solutions with periods close to $T$ in every neighbourhood $\mathscr{U} \backslash\{0\}$ of the equilibrium point:

$$
\text { \#\{periodic solutions with periods near } T \text { in } \mathscr{U} \backslash\{0\}\} \geq|\sigma| \text {. }
$$

We formulate this result more precisely; it prescribes the periods of the required periodic solutions and not their energies, in contrast to the existence results mentioned before.

TheOREM. Assume that $\mathbb{R}^{2 n} \in E \oplus F$ is an invariant splitting under $A$, so that all solutions of (1.2) in E have period $T>0$ while none of the solutions in $F \backslash\{0\}$ has this period. Assume, moreover, that $H_{x x}(0) \mid E$ is non-degenerate and denote its signature by $2 \sigma$.

If $\{0\}$ is an isolated T-periodic solution, there exist two non-negative integers $k_{+}$and $k_{-}$with

$$
k_{+}+k_{-} \geq|\sigma|=\left|n_{+}-n_{-}\right|
$$


such that for every neighbourhood $\mathscr{U} \backslash\{0\}$ the following holds true: for every $\tau \epsilon$ $[T-\varepsilon, T+\varepsilon]$ the number of $\tau$-periodic solutions of $(1.1)$ in $\mathcal{U} \backslash\{0\}$ is at least $k_{+}$if $\tau>T$ and at least $k_{-}$if $\tau<T$. Here $\varepsilon>0$ is a positive number depending on $U$.

In order to outline the proof, we recall that there is a classical variational principle for which the critical points are the required periodic solutions. Using the well known Lyapunov-Schmidt reduction procedure, Fadell and Rabinowitz in their proof reduce the problem of finding the critical points to that of finding the critical points of a related function, say $\mathrm{g}$, which is defined on the finite-dimensional space $E$. This function is, moreover, invariant under an action of the group $G=S^{1}$, which stems from the fact that the Hamiltonian system is independent of time. The critical points of $g$ are then found by means of mini-max techniques based on a cohomological index theory for $S^{1}$-actions, generalizing earlier techniques based on the notion of genus or category.

Our approach uses a different idea. We shall study the flow $\dot{x}=-\nabla g(x)$ of the reduced functional using an equivariant version of Conley's homotopy index theory, which was introduced in [5] and has already turned out to be useful in finding global fixed points of symplectic maps.

To an isolated invariant set $S$ of the gradient flow, which is also invariant under the action of the group $G$, we associate a cohomological index $I_{G(S)}^{*}$ which is an $R$-module over the ring $R=H_{G}^{*}(\{0\}) \simeq H^{*}\left(\mathbb{C} P^{\infty}\right)$. This index has the property to be invariant under continuation. The required critical $G$-orbits constitute a Morse decomposition of the set $S$ of bounded solutions in a given neighbourhood of the origin $\{0\}$. The origin is a $G$-fixed point and corresponds to the trivial periodic solution at the equilibrium point. Those sets in the Morse filtration of $S$ which contain the fixed point of the action contain a free $R$-submodule in their cohomological index. The dimension of its generator adds information additional to the cohomological index of Fadell and Rabinowitz. In fact it allows us to estimate also the number of critical orbits in the presence of a $G$-fixed point set and makes the existence proof algebraic in nature.

We merely illustrate the idea in the special case at hand; the general equivariant Morse theory will appear elsewhere.

\section{The Lyapunov-Schmidt reduction}

In this section we recall the Lyapunov-Schmidt reduction from [1], to which we also refer for the details.

Normalizing the period to 1 , we look for 1-periodic solutions of the equation

$$
\dot{x}=\lambda J \nabla H(x), \quad x(0)=x(1)
$$

for a constant $\lambda$ in the neighbourhood of $\lambda=\mu$, where $\mu=T$. A solution $x(t)$ of (2.1) corresponds to the $\lambda$-periodic solution $y(t):=x\left(\lambda^{-1} t\right)$ of (1.1).

With the Hilbert spaces $H:=H_{1}\left(S^{1}, \mathbb{R}^{2 n}\right)$ and $L=L_{2}\left(S^{1}, \mathbb{R}^{2 n}\right)$ we define the $C^{1}$-mapping

$$
F_{\lambda}: H \rightarrow L: x \rightarrow J \dot{x}+\lambda \nabla H(x) .
$$

Its zeros are the required periodic solutions of $(2.1)$, since $J^{2}=-\mathrm{id}$. By assumption the plane $E$ contains all the $T$-periodic solutions of the linearized system at the 
origin; we therefore conclude for the derivative $d\left(F_{\lambda}\right)(0)$, if $\lambda=\mu$,

$$
\text { kernel } d\left(F_{\mu}\right)(0)=: N \cong E \text {. }
$$

Since the cokernel coincides with $N$, the operator $d\left(F_{\mu}\right)(0)$ is a Fredholm operator of index 0 . Denote by $\pi$ and $\pi^{\perp}$ the orthogonal projections (with respect to the $L_{2}$ inner product) onto $N$ and onto its complement. Then the map

$$
H \times \mathbb{R} \rightarrow N^{\perp}, \quad(z, \lambda) \rightarrow \pi^{\perp} \circ F_{\lambda}(z)
$$

is a $C^{1}$-mapping whose derivative in $z$ is, at $(z, \lambda)=(0, \mu)$, a surjective Fredholm operator with kernel $N \times \mathbb{R}$. The implicit function theorem implies the existence of a $C^{1}$-mapping

$$
\psi: N \times \mathbb{R} \rightarrow N^{\perp}
$$

defined on an open neighbourhood $B \times V$ of $(z, \lambda)=(0, \mu)$ and satisfying $\pi^{\perp} \circ F_{\lambda}(x+$ $\psi(x, \lambda))=0$ for $(x, \lambda) \in B \times V$. It therefore remains to solve the equation

$$
\pi \circ F_{\lambda}(x+\psi(x, \lambda))=0 \quad \text { for }(x, \lambda) \in B \times V \subset N \times \mathbb{R} .
$$

In order to study the solutions of (2.6), we make use of the variational structure of the problem. One verifies easily that $z \in H$ satisfies the equation $F_{\lambda}(z)=0$ if and only if it is a critical point of the function $f_{\lambda}: H \rightarrow \mathbb{R}$ defined by

$$
f_{\lambda}(z)=\int_{0}^{1}\left\{\frac{1}{2}\langle z, J \dot{z}\rangle+\lambda H(z)\right\} d t .
$$

Abbreviating $\varphi_{\lambda}(x)=x+\psi(x, \lambda)$, we introduce the reduced functional

$$
a_{\lambda}(x)=f_{\lambda} \circ \varphi_{\lambda}(x), \quad x \in N .
$$

One verifies readily that $x$ is a solution of (2.6) if and only if it is a critical point of $a_{\lambda}$. It remains to find critical points of $a_{\lambda}$ defined on $B \subset N$ other than the origin $\{0\}$,

$$
\nabla a_{\lambda}(0)=0,
$$

which corresponds to the trivial periodic solution. Moreover, near $x=0$ (with a quadratic form $Q$ )

$$
\begin{aligned}
a_{\lambda}(x) & =(\lambda-\mu) \frac{1}{2} Q(x)+o\left(|x|^{2}\right) \\
& =(\lambda-\mu) \frac{1}{2}\left\langle H_{x x}(0) x(0), x(0)\right\rangle+o\left(|x|^{2}\right) .
\end{aligned}
$$

If $\lambda \neq \mu$, the critical point $x=0$ is therefore, by our assumption on $H_{x x}(0) \mid E$, a hyperbolic critical point having Morse index $2 n_{+}$if $\lambda>\mu$ and $2 n_{-}$if $\lambda<\mu$. Since, again by assumption, $2 n_{+} \neq 2 n_{-}$, one concludes easily a critical point of $a_{\lambda}$ other than $x=0$ either for $\lambda>\mu$ or $\lambda<\mu$. However, to find more critical points the $G$-action is crucial.

Define the $G$-action, $G=S^{1}$, on $H$ by

$$
L_{\theta} z(t)=z(t+\theta), \quad \theta \in \mathbb{R} / \mathbb{Z}=S^{1} .
$$

From the time independence of the Hamiltonian one concludes that $f_{\lambda}\left(L_{\theta} z\right)=f_{\lambda}(z)$ for $z \in H$, from which it also follows that the function $a_{\lambda}$ is invariant under this action:

$$
a_{\lambda}\left(L_{\theta} x\right)=a_{\lambda}(x), \quad x \in N .
$$

Moreover, when restricted to $N$, the group action $(2.11)$ has the origin $\{0\}$ as the only fixed point. 
Summarizing, it remains to find equilibrium points other than $x=0$ of the gradient flow

$$
\dot{x}=-\nabla a_{\lambda}(x) \quad \text { on } B \subset N,
$$

which is equivariant with respect to a group action of $G=S^{1}$ whose only fixed point is the origin. Moreover, in a neighbourhood of the origin $a_{\lambda}$ is of the form (2.10).

Since we assume in the following that the origin in $\mathbb{R}^{2 n}$ is an isolated $T$-periodic solution of (1.1), we also know that $x=0$ is an isolated critical point of the function $a_{\lambda}$ for $\lambda=\mu$.

\section{Isolated invariant sets and the cohomological index}

We denote by $\varphi_{\lambda}^{\prime}$ the family of flows of the gradient equation (2.13):

$$
\frac{d}{d t} \varphi_{\lambda}^{\prime}=-\nabla a_{\lambda} \circ \varphi_{\lambda}^{t}, \quad \varphi_{\lambda}^{0}=\mathrm{id}
$$

on $B \subset N$, and recall first some concepts of Conley's homotopy index theory from $[1],[2]$ and [5].

If $Q \subset B$ is a compact set, the maximal invariant set under the flow $\varphi_{\lambda}$ in $Q$ is given by

$$
S_{\lambda}=S_{\lambda}(Q)=\left\{x \in Q \mid \varphi_{\lambda}^{t}(x) \in Q \text { for all } t \in \mathbb{R}\right\} .
$$

If the set $S_{\lambda}(Q)$ is contained in the interior of $Q$, it is called an isolated invariant set and $Q$ is called an isolating set.

For an isolated invariant set $S_{\lambda}$ there exists an index pair $(X, A)$ such that $H^{*}(X, A)$ does not depend on the particular choice of the index pair.

We now make use of the group action $G$ and consider an isolated invariant set $S$ which is also $G$-invariant; then we find a $G$-invariant index pair for $S$ by averaging an index pair $(X, A)$ over the group $G$ :

$$
(G X, G A)=\bigcup_{g \in G}(g \cdot X, g \cdot A),
$$

so that $G$-invariant index pairs do exist for any $G$-invariant and isolated invariant set $S$. If $(X, A)$ is a $G$-invariant index pair for such a set $S=S_{\lambda}$, we define an equivariant cohomological index

$$
I_{\lambda}^{*}(S)=H_{G}^{*}(X, A),
$$

where $H_{G}^{*}$ is the equivariant Alexander cohomology with respect to the operation of $G$ with real coefficients. It is defined as the ordinary Alexander cohomology

$$
H_{G}^{*}(X, A)=H^{*}\left(X \times E_{/ G}, A \times E_{/ G}\right),
$$

where $E$ is a contractible topological space with a free continuous $G$-action and where the operation of $G$ on the product is diagonal: $g \cdot(x, e)=(g \cdot x, g \cdot e)$ for $g \in G$. We note that $I_{\lambda}^{*}(S)$ becomes a module of the ring

$$
\left.R=H_{G}^{*}\{0\}\right) \cong H^{*}\left(E_{/ G}\right)
$$

as follows. We denote the $G$-equivariant retraction of $N$ onto the $G$-invariant set consisting of the origin $\{0\}$ in $N$ by

$$
r: N \rightarrow\{0\}
$$


The operation of $H_{G}^{*}(\{0\})$ on $I_{\lambda}^{*}(S)=H_{G}^{*}(X, A)$ is then defined by

$$
\begin{gathered}
H_{G}^{*}(\{0\}) \times H_{G}^{*}(X, A) \rightarrow H_{G}^{*}(X, A), \\
(\alpha, u) \rightarrow \alpha * u=\left(r_{\mid X}\right)^{*} \alpha \cup u,
\end{gathered}
$$

where $\cup$ denotes the equivariant cup product. In our special case in which $G=S^{1}$ we have $E_{/ G} \cong \mathbb{C P}^{\infty}$. Therefore

$$
R=\mathbb{R}[\omega], \quad \operatorname{dim} \omega=2
$$

is the polynomial ring in one variable with real coefficients.

It is proved in theorem 1 of [5] that this module structure of $I_{\lambda}^{*}(S)$ does not depend on the choice of the index pair but is an invariant of the set $S$ alone.

\section{Proof of the theorem}

LEMMA 1. For every $\lambda \in V$ the origin $x=\{0\}$ is a $G$-invariant and isolated invariant set of the flow $\varphi_{\lambda}$. If $\lambda \neq \mu$, there exists a $u_{\lambda} \in I_{\lambda}^{*}(\{0\})$ such that the map

$$
R \rightarrow I_{\lambda}^{*}(\{0\}): \alpha \rightarrow \alpha * u_{\lambda}
$$

is an isomorphism. Moreover,

$$
\begin{array}{ll}
u_{\lambda} \in I_{\lambda}^{2 n^{+}}(\{0\}) & \text { if } \lambda>\mu, \\
u_{\lambda} \in I_{\lambda}^{2 n}-(\{0\}) & \text { if } \lambda<\mu .
\end{array}
$$

Proof. Any isolated critical point of a function is an isolated invariant set of the gradient flow as one verifies easily. For $\lambda=\mu$ the origin $\{0\}$ is an isolated critical point of $a_{\mu}$ by assumption. For $\lambda \neq \mu$ it is, in view of (2.10), even a non-degenerate critical point of $a_{\lambda}$ having Morse index $2 n_{+}$for $\lambda>\mu$ and $2 n_{-}$for $\lambda<\mu$. It is therefore a hyperbolic invariant set of the gradient flow. With the equivariant retraction (3.7) onto this hyperbolic invariant set we conclude from proposition 1 of [5] that there is indeed a $u_{\lambda} \in I^{2 n_{+}}(\{0\})$ if $\lambda>\mu$ and $u_{\lambda} \in I^{2 n_{-}}(\{0\})$ if $\lambda<\mu$ such that the map

$$
H_{G}^{*}(\{0\}) \rightarrow I_{\lambda}^{*}(\{0\}): \alpha \rightarrow \alpha * u_{\lambda}=r^{*} \alpha \cup u_{\lambda}
$$

is an isomorphism of Abelian groups as claimed in the lemma.

The most useful and crucial property of the cohomological index $I_{\lambda}^{*}\left(S_{\lambda}\right)$ is its invariance under deformations of the flow. This is in fact the main point in Conley's definition of an index pair. In the equivariant case the proof can be found in theorem 1 of [5]. In particular if $\lambda_{1}$ is near $\lambda$, then there is an $R$-isomorphism $I_{\lambda}^{*}\left(S_{\lambda}\right) \rightarrow I_{\lambda_{1}}^{*}\left(S_{\lambda_{1}}\right)$. From this we obtain the following:

LEMMA 2. If $Q$ is a G-invariant isolating neighbourhood of $x=0$ in $B$ with $S_{\mu}(Q)=\{0\}$, then there is a neighbourhood $\mathcal{U}_{1}(\mu) \subset V$ of $\mu$ in $\mathbb{R}$ so that $Q$ is isolating for the flows $\varphi_{\lambda}$ for all $\lambda \in U_{1}(\mu)$. Moreover, there exist isomorphisms of $R$-modules

$$
I_{\lambda}^{*}\left(S_{\lambda}\right) \cong I_{\mu}^{*}(\{0\})
$$

with $S_{\lambda}=S_{\lambda}(Q)$. 
Recall next the definition of a Morse decomposition.

Definition. A Morse decomposition of an isolated invariant set $S$ is a finite collection $\left\{M_{p}\right\}, p \in P$ of disjoint compact and invariant subsets $M_{p} \subset S$ which can be ordered $\left\{M_{1}, \ldots, M_{k}\right\}$ such that for all $x \in S \backslash \cup M_{p}$ there is a pair $i<j$ of indices satisfying

$$
\omega(x) \in M_{i}, \quad \omega^{*}(x) \in M_{j},
$$

where $\omega$ (resp. $\omega^{*}$ ) denotes the positive (resp. negative) limit set of $x$.

We consider in the following the isolated invariant and $G$-invariant set $S_{\lambda}=S_{\lambda}(Q)$ of lemma 2. It suffices, moreover, to consider those values of $\lambda$ for which $S_{\lambda}$ contains only a finite number $k=k_{\lambda}$ of critical orbits. A critical orbit is necessarily a $G$-invariant and isolated invariant set of the flow. We can order the critical orbits $\mathcal{O}_{j}$ in $S_{\lambda}$ by setting

$$
a_{\lambda}\left(\mathscr{O}_{l}\right) \geq a_{\lambda}\left(\mathscr{O}_{k}\right) \quad \text { for } l \geq k
$$

so that the critical orbits

$$
\left\{O_{0}, O_{1}, \ldots, O_{k}\right\}, \quad k=k_{\lambda}
$$

constitute an ordered Morse decomposition of $S_{\lambda}$. Define, moreover, a filtration of $S_{\lambda}$

$$
S_{\lambda}^{0} \subset S_{\lambda}^{1} \subset \cdots \subset S_{\lambda}^{k}=S_{\lambda},
$$

consisting of $G$-invariant isolated invariant sets as follows:

$$
S_{\lambda}^{p}=\left\{x \in S_{\lambda} \mid \omega(x) \text { and } \omega^{*}(x) \subset \mathscr{O}_{0} \cup \mathcal{O}_{1} \cup \cdots \cup \mathcal{O}_{p}\right\} .
$$

In particular $\left\{S^{p-1}, \mathscr{O}_{p}\right\}$ is an ordered Morse decomposition of $S^{p}$ and the cohomological indices of the sets $S^{p}$ are related to those of the critical orbits $O_{p}$ as follows:

LEMmA 3. There is an exact triangle of $R$-module homomorphisms

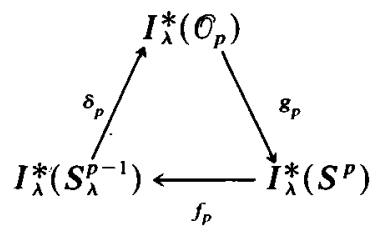

where $f_{p}$ and $g_{p}$ are of degree 0 and where $\delta_{p}$ is of degree 1 , i.e. raises the dimension by 1 .

Proof. There exists, by theorem 3.1 in [2], compact sets $X_{1} \subset X_{2} \subset X_{3}$ in $Q$ such that $\left(X_{3}, X_{2}\right)$ is an index pair of $\mathcal{O}_{p},\left(X_{2}, X_{1}\right)$ is an index pair of $S^{p-1}$ and $\left(X_{3}, X_{1}\right)$ is an index pair of $S^{p}$. We can easily adapt the construction to the equivariant case such that the compact sets $X_{j}$ are, in addition, $G$-invariant by replacing each $X_{j}$ by $G X_{j}=\left\{g \cdot x \mid g \in G\right.$ and $\left.x \in X_{j}\right\}$. The lemma then follows from the long exact sequence for the triple of sets

$$
\hat{X}_{3} \supset \hat{X}_{2} \supset \hat{X}_{1}
$$

where

$$
\hat{X}_{j}=\left(X_{j} \times E\right)_{/ G},
$$


together with the following observations: the maps $f_{p}$ and $g_{p}$ are $R$-module homomorphisms since they are inclusion-induced. In fact, it suffices to show that the equivariant cup product is natural with respect to equivariant maps. This statement can be reduced to the naturality property of the ordinary cup product (see 5.6.8 of [9]). Also $\delta_{p}$ is an $R$-module homomorphism. In order to see this, we apply 5.6.12 of [9] to the excisive (see p. 188 of [9]) couple of pairs $\left\{\left(X_{3}, X_{2}\right)_{G},\left(X_{2}, X_{1}\right)_{G}\right\}$. For $u \in H_{G}^{*}\left(X_{2}, X_{1}\right)=I^{*}\left(S^{p-1}\right)$ we obtain

$$
\delta^{*}(\alpha * u)=\delta^{*}\left(u \cup\left(r_{\mid X_{3}}\right)^{*} \alpha\right)=\left(\delta^{*} u\right) \cup\left(r_{\mid X_{2}}\right)^{*} \alpha=\alpha * \delta^{*}(u) .
$$

This completes the proof of the lemma.

In order to make use of lemma 3, we need some information about the cohomological indices of the isolated critical orbits $\sigma_{p}$. Since we do not assume the orbits to be non-degenerate, we cannot calculate $I_{\lambda}^{*}\left(O_{p}\right)$ explicitly. The following statement however will be sufficient for our purpose:

LEMMA 4. Assume $O$ is an isolated critical orbit which is not a fixed point for the action of $G$. Then $I_{\lambda}^{*}(\mathcal{O})$ is a trivial $R$-module in the sense that for every $u \in I_{\lambda}^{*}(\mathcal{O})$ and every $\alpha \in R$ with $\operatorname{dim} \alpha>0$ we have

$$
\alpha * u=0 .
$$

Proof. By assumption, $\mathcal{O}$ is not a $G$-fixed point since $G=S^{1}$; the isotropy groups in $O$ are therefore finite and, since we are using real coefficients, there is an isomorphism

$$
H_{G}^{*}(\mathcal{O}) \rightarrow H^{*}\left(\mathcal{O}_{/ G}\right)=H^{*}(\{\text { point }\})
$$

Indeed this is lemma 6.11 in [3]. Assume now $\alpha * u \neq 0$ for some $u \in I_{\lambda}^{*}(\mathcal{O})$ and $\alpha \in R$ with $\operatorname{dim} \alpha>0$. Then it follows from proposition 2 in [5] that $\left(r_{10}\right)^{*} \alpha \neq 0$ in $H_{G}^{*}(O)$, contradicting the statement (4.9). Consequently $\alpha * u=0$ as claimed.

This statement will allow us to estimate the number $k_{\lambda}$ of critical orbits in $S_{\lambda}$ by purely algebraic means. We shall prove:

LEMMA 5. There is an integer $m$, which is independent of $\lambda$, such that for $\lambda \neq \mu$ the number of critical orbits in $S_{\lambda}$ can be estimated by

$$
k_{\lambda} \geq\left|m-\frac{1}{2} \operatorname{dim} u_{\lambda}\right|
$$

where $u_{\lambda}$ is the generator of the free $R$-module $I_{\lambda}^{*}(\{0\})$ in lemma 1 .

Postponing the proof, we first show that the theorem follows. From lemma 1 we know that $\operatorname{dim} u_{\lambda}=2 n_{+}$if $\lambda>\mu$ and $\operatorname{dim} u_{\lambda}=2 n_{-}$if $\lambda<\mu$. Setting $k^{+}=k_{\lambda}$ for $\lambda>\mu$ and $k^{-}=k_{\lambda}$ for $\lambda<\mu$, we therefore conclude from (4.10) that indeed $k^{+}+k^{-} \geq$ $\left|n_{+}-n_{-}\right|=|\sigma|$.

For the proof of lemma 5 we need some further notation. For any $R$-module $M$ we define the torsion submodule by

$$
\tau(M):=\{u \in M \mid \alpha * u=0 \text { for some } \alpha \in R\}
$$

and abbreviate the quotient

$$
\Phi(M):=M_{/ \tau(M)},
$$


which is a free $R$-module, i.e.

$$
\tau(\Phi[M])=0 .
$$

Recall now that the critical orbits $\left\{\mathcal{O}_{j}\right\}$ for $0 \leq j \leq k$ with $k=k_{\lambda}$ constitute an ordered Morse decomposition of the isolated invariant set $S_{\lambda}$; recall also the filtration $S_{\lambda}^{0} \subset S_{\lambda}^{1} \subset \cdots \subset S_{\lambda}^{k}=S_{\lambda}$.

Let the index $l$ correspond to the distinguished critical orbit at the origin

$$
\sigma_{1}=\{0\}
$$

which is a fixed point of the action $G$.

In order to prove lemma 5 we shall study the quotients $\Phi\left[I_{\lambda}^{*}\left(S_{\lambda}^{j}\right)\right]$ for $0 \leq j \leq k$ and claim:

LEMMA 6

$$
\Phi\left[I_{\lambda}^{*}\left(S_{\lambda}^{p}\right)\right]=0 \quad \text { for } 0 \leq p<l ;
$$

moreover it vanishes also in odd dimensions for $l \leq p \leq k$.

(ii) Denote by $m_{p}$ half the minimal dimension for which $\Phi\left[I^{*} \lambda\left(S_{\lambda}^{p}\right)\right]$ does not vanish; then

$$
\begin{gathered}
\frac{1}{2} \operatorname{dim} u_{\lambda}-l \leq m_{l} \leq \frac{1}{2} \operatorname{dim} u_{\lambda} \quad \text { for } p=l . \\
m_{p-1} \leq m_{p} \leq m_{p-1}+1 \quad \text { for } l<p \leq k .
\end{gathered}
$$

Postponing the proof of the lemma, we first deduce lemma 5 from lemma 6.

If $\frac{1}{2} \operatorname{dim} u_{\lambda} \geq m_{k}$, then in view of (ii) and (iii) we have $m_{k} \geq m_{l} \geq \frac{1}{2} \operatorname{dim} u_{\lambda}-l$, so that

$$
k \geq l \geq \frac{1}{2} \operatorname{dim} u_{\lambda}-m_{k} \text {. }
$$

If on the other hand $m_{k} \geq \frac{1}{2} \operatorname{dim} u_{\lambda}$, then in view of (iii) we find that $m_{k} \leq m_{l}+(k-l)$, so that by (ii)

$$
k \geq m_{k}-m_{l}+l \geq m_{k}+l-\frac{1}{2} \operatorname{dim} u_{\lambda} .
$$

Combining the two estimates for $k$, we find

$$
k \geq\left|m_{k}-\frac{1}{2} \operatorname{dim} u_{\lambda}\right| \text {. }
$$

Recall now that for $j=k$ the integer $m_{k}$ does not depend on $\lambda$ since it is obtained from the isomorphism class of $I_{\lambda}^{*}\left(S_{\lambda}\right)$, which is isomorphic to $I_{\mu}^{*}(\{0\})$ in view of lemma 1 and therefore independent of $\lambda$. Setting $m:=m_{k}$, the statement of lemma 5 follows from (4.18).

It finally remains to prove lemma 6 which we do by induction. We first prove that

$$
\omega^{p+1} * I_{\lambda}^{*}\left(S_{\lambda}^{p}\right)=0 \quad \text { for } 0 \leq p<l,
$$

where $\omega$ generates the ring $R=\mathbb{R}[\omega]$ and $\operatorname{dim} \omega=2$. In view of lemma 4 the statement (4.19) is true for $p=0<l$, since $S_{\lambda}^{0}=\mathcal{O}_{0}$ does not contain any fixed point of the action of $G$. Moreover, if (4.19) is true for $S^{p-1}$ with $0<p<l$, then it also is true for $S^{p}$. To see this, consider the exact triangle (4.7).

For any $u \in I_{\lambda}^{*}\left(S^{p}\right)$ we have $f_{p}\left(\omega^{p} * u\right)=\omega^{p} * f_{p}(u)=0$ in $I_{\lambda}^{*}\left(S^{p-1}\right)$ by induction assumption. Hence by exactness we have $\omega^{p} * u=g_{p}(v)$ for some $v \in I_{\lambda}^{*}\left(\sigma_{p}\right)$, but $\omega * v=0$ in view of lemma 4 . Consequently $\omega^{p+1} * u=\omega * g_{p}(v)=g_{p}(\omega * v)=0$, proving the claim (4.19). 
Consider next the exact triangle (4.7) for $p=l$, i.e.

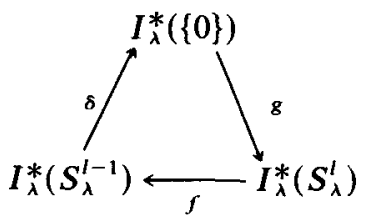

We have shown in (4.19) that $\Phi\left[I_{\lambda}^{*}\left(S^{l-1}\right)\right]=0$, and since in view of lemma $1 I_{\lambda}^{*}(\{0\})$ is a free $R$-module generated by $u_{\lambda}$, we conclude that $\delta=0$ and $g$ is injective. This implies that $2 m_{1} \leq \operatorname{dim} u_{\lambda}$. Moreover, if $u$ is a non-torsion element in $I_{\lambda}^{*}\left(S^{\prime}\right)$ with $\operatorname{dim} u=2 m_{l}$, then

$$
f\left(\omega^{\prime} * u\right)=\omega^{\prime} * f(u)=0
$$

by (4.19), so that $\omega^{I} * u=g(x)$ for some $x \in I_{\lambda}^{*}(\{0\})$ by exactness of (4.20). Since $I_{\lambda}^{*}(\{0\})$ vanishes in odd dimensions, we therefore conclude that $\operatorname{dim}\left(\omega^{\prime} * u\right)$ is even, and since $I_{\lambda}^{*}(\{0\})$ is generated by $u_{\lambda}$, we also have

$$
\operatorname{dim}\left(\omega^{\prime} * u\right)=2 l+\operatorname{dim} u \geq \operatorname{dim}\left(u_{\lambda}\right)
$$

so that $m_{l}=\frac{1}{2} \operatorname{dim} u \geq \frac{1}{2} \operatorname{dim} u_{\lambda}-l$.

Now let $l<p \leq k$ and assume the statements of lemma 6 to hold true for $S^{p-1}=$ $S_{\lambda}^{p-1}$. Consider again the exact triangle

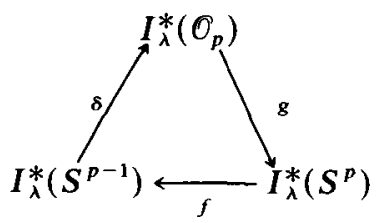

For every class $0 \neq[u] \in \Phi\left[I_{\lambda}^{*}\left(S^{p}\right)\right]$ we conclude $0 \neq[f(u)] \in \Phi\left[I_{\lambda}^{*}\left(S^{p-1}\right)\right]$ since $I_{\lambda}^{*}\left(O_{p}\right)$ is of pure torsion in view of lemma 4. This shows, using the induction assumption, that $\operatorname{dim} u$ is even and that $2 m_{p} \geq 2 m_{p-1}$. If $v \in I_{\lambda}^{*}\left(S^{p-1}\right)$ is a non-torsion element with $\operatorname{dim} v=2 m_{p-1}$, then

$$
\delta(\omega * v)=\omega * \delta(v)=0 \quad \text { in } I_{\lambda}^{*}\left(O_{p}\right)
$$

by lemma 4. By exactness there is a non-torsion element $u$ in $I_{\lambda}^{*}\left(S^{p}\right)$ satisfying $f(u)=\omega * v$, so that $\operatorname{dim} u=2+2 m_{p-1}$ and we conclude that $m_{p} \leq m_{p-1}+1$. This finishes the proof of lemma 6.

\section{REFERENCES}

[1] C. Conley. Isolated Invariant Sets and the Morse Index. CBMS Regional Conference Series in Mathematics 38. AMS, Providence (1978).

[2] C. Conley \& E. Zehnder. Morse type index theory for flows and periodic solutions for Hamiltonian equations. Comm. Pure Appl. Math. 37 (1984), 207-253.

[3] E. Fadell \& P. Rabinowitz. Generalized cohomological index theories for Lie group actions with an application to bifurcation questions for Hamiltonian systems. Invent. Math. 45 (1978), 139-174.

[4] A. Floer. Proof of the Arnold Conjecture for surfaces and generalizations to certain Kählermanifolds. Duke Math. J. 53 (1986), 1-32. 
[5] A. Floer. A refinement of the Conley index and an application to the stability of hyperbolic invariant sets. Ergod. Th. \& Dynam. Sys. 7 (1987), 93-103.

[6] A. Lyapunov. Problème général de la stabilité des mouvements. Ann. Fac. Sci. Toulouse 2 (1907), 203-474.

[7] J. Moser. Periodic orbits near an equilibrium and a theorem by A. Weinstein. Comm. Pure Appl. Math. 29 (1976), 727-747.

[8] J. Moser. Addendum to 'Periodic orbits near an equilibrium and a theorem by A. Weinstein'. Comm. Pure Appl. Math. 31 (1978), 529-530.

[9] E. Spanier. Algebraic Topology. McGraw-Hill, New York (1966).

[10] A. Weinstein. Normal modes for nonlinear Hamiltonian systems. Invent. Math. 20 (1973), 47-57. 